\title{
Parabrachial neuron development: Effects of pre- and neonatal undernutrition in the rat
}

\author{
Carmen Torrero, Mirelta Regalado, Lorena Rubio, Manuel Salas
}

Department of Developmental Neurobiology and Neurophysiology, Institute of Neurobiology, University of Mexico, Queretaro, Mexico

Email: masal@unam.mx

Received 12 September 2012; revised 15 October 2012; accepted 25 October 2012

\begin{abstract}
Pre-and neonatal fasting in the rat has been used as an experimental model to obtain information on how the newborn gustatory system can be damaged, interfering with the basic sensory and hedonic processes to different tastants. Fasting during the prenatal period and for 24 days postnatally results in significant reductions of body and brain weight, number of branches, dendritic density, and cross-sectional area of the PBN multipolar neurons in the central lateral and central medial subnucleus particularly at postnatal days 20 and 30. Furthermore, the underfeeding paradigm affected more the middle portions of the dendritic tree than other parts of the neurons possibly disturbing the afferent characteristics of neuronal activity propagation that may partly disrupt the elaboration of synaptic plasticity at later ages. These findings may play a role in the development of complex physiological phenomena such as food intake, taste discrimination, learning taste aversion, and appetitive behavior.
\end{abstract}

Keywords: Parabrachial Neurons; Development; Brainstem; Undernutrition; Rats

\section{INTRODUCTION}

Several lines of evidence have shown that the rodent parabrachial nucleus (PBN) is a brain stem complex of polysensory neuronal subnuclei that receive and integrate various ascending and descending inputs. These inputs participate in generating the sensory and hedonic aspects of taste, in the plastic changes associated with experience, and in a variety of sensory, visceral functions, such as the modulation of gastrointestinal, cardiovascular and respiratory systems [1-10].

The representation of hedonics and quality of taste cues in the rat PBN has been studied by measuring the c-fos immunoreactivity evoked by oral gustatory stimuli with different qualities and different hedonic values.
Thus, the rostral External Lateral Subnucleus (ELS) is related to general visceral inputs; the caudal or External Medial Subnucleus (EMS) is associated with negative hedonics or aversive behavior; and the Dorsal Lateral Subnucleus (DLS) is related to positive hedonics or ingestive behavior. Taste information of palatable sucrose and $\mathrm{NaCl}$ projects to the external Central Lateral Subnucleus (CLS) and Central Medial Subnucleus (CMS), respectively [5].

In the rat, different perinatal underfeeding protocols are associated with severe alterations in the sensory neuroanatomical and functional organization as revealed by delayed ear- and eye-opening, and alterations of olfactory, auditory and visual electrophysiological properties, and sensorimotor behavioral performance [11-15]. The most common anatomical changes indicate a clear neuronal hypoplasia accompanied by poor dendritic arborizations and spines distribution that play a role in integrating and processing of the patterns of ascending information from the sensory relays and the limbic, cerebral, and cerebellar cortices, structures which undergo intense neonatal cell proliferation [16-22]. Although there is considerable knowledge about the anatomical and functional deficiencies of the gustatory system in early underfed rats, information on the alterations occurring in the PBN at critical stages of life is lacking.

The present study analyzes the effects of perinatal food restriction upon the neuronal development in the multipolar PBN, particularly at the central lateral (CLS) and central medial (CMS) subnuclei functional segregation that is closely associated with the sucrose and $\mathrm{NaCl}$ afferent projections in route to the cerebral cortex [5].

\section{MATERIAL AND METHODS}

\subsection{Animals}

Subjects were Wistar rats $(250 \mathrm{~g}-300 \mathrm{~g})$, descendants of a stock originally purchased from Harlan Sprague-Dawley, INC., and they were maintained in an automatically controlled room at $22^{\circ} \mathrm{C} \pm 2{ }^{\circ} \mathrm{C}$, humidity $(50 \%)$, on a light/ dark cycle (lights on at 07:00), with water and food $(\mathrm{Pu}-$ 
rina chow) ad libitum. For mating, a male was placed in a plastic cage containing three virgin females $(200 \mathrm{~g}$ $250 \mathrm{~g}$ ). Sperm-positive females were placed individually in plastic maternity cages $\left(50 \times 40 \times 20 \mathrm{~cm}^{3}\right)$ with wood shavings as nesting material. The day after birth, pups were weighed and sexed, and four females and four males from each litter were randomly distributed among dams in order to minimize genetic and nutritional differences that may influence the experimental results. The distinctive bilateral thoracic and abdominal line of nipples and the shorter anogenital distance in the females were used as criteria for sex recognition [23].

\subsection{Perinatal Underfeeding Procedure}

The undernourished male subjects (UG) used in this group $(\mathrm{n}=18)$ came from at least eight different litters. The normal chow diet requirement was calculated by measuring the food intake of a group of 6 pregnant control rats $(230 \mathrm{~g}-250 \mathrm{~g})$ every week during a 21-day period. The resulting average food intake for each week was the basal level used to calculate the food-intake percentage of the UG females.Thus, dams were fed from G6 to G12 with $50 \%$ (7.8 g) of the normal diet (Purina chow), from G13 to G19 with $70 \%(10.9 \mathrm{~g})$, and with $100 \%(15.6 \mathrm{~g})$ of the same diet until parturition to avoid resorption or cannibalism of pups. This protocol was chosen because neurogenesis in the inner division of the external lateral and the external medial subnuclei of the PBN and afferent connectivity occur primarily from E16 to E21 [24]. At birth, prenatally underfed newborns were nursed by two gestationally underfed dams, in one of which the main galactophorous ducts had been tied subcutaneously [25]. To continue the neonatal underfeeding paradigm, these two lactating dams were interchanged every $12 \mathrm{~h}$ between litters from postnatal days 1 to 24 . This crossfostering procedure attenuates the effects on the pups of maternal sensory deprivation. After this, the older group of pups ( 30 days old) had free access to solid food (Purina chow), so they had a 6-day period of dietary rehabilitation. No attempts were made to measure food intake in pregnant dams or newborn rats. Approximately $80 \%$ of the total underfed subjects included here were undernourished during the light phase of the cycle.

\subsection{Control Group (CG)}

The CG of animals consisted of 18 male pups obtained from eight normally nourished subjects, nursed by wellfed mothers with free access to food and water. After birth, pups were fed and handled by interchanging a pair of normally lactating mothers every 12 hours as previously described [26]. After weaning on day 25, subjects had free access to water and solid food. To evaluate the effects of the nutritional paradigms on physical growth, body and brain weights of subjects with different experimental treatments were noted.

\subsection{Histology}

A total of 36 male rats were subjected to two dietary treatments ( $n=18, \mathrm{UG}$ and $n=18, \mathrm{CG})$, each with three age groups. They were weighed, deeply anesthetized with ether, and sacrificed by decapitation at 12,20 , and 30 days of age, resulting in six subjects per age and treatment. The brains were immediately removed and weighed wet, cut into three coronal blocks, and immersed in a Golgi-Cox solution for impregnation. Three weeks later, the blocks were dehydrated and embedded in low viscosity nitrocellulose. Subsequently, they were cut in coronal sections of $120-150 \mu \mathrm{m}$ and mounted serially. The slides were coded to ensure blind evaluation with respect to age and dietary treatment of subjects. Furthermore, during the neuronal image digitizing, the experimenter had access only to the code numbers and not to the ages and experimental conditions of the brain material. Identification and location of the PBN complex were based on Paxinos and Watson's atlas [27]. Anteriorposterior coordinates for the localization of the PBN corresponded to values ranging from Bregma -9.68 to $-9.80 \mathrm{~mm}$.

\subsection{Morphometric Measurements}

The present study was based on a total of 540 well-impregnated, multipolar neurons underlying the gustatory relay whose dendritic field was confined to one section, as evidenced by light microscopy in each experimental condition, age group, and neuronal parameter (Figure 1). Dendritic arbor measurements were obtained by counting the number of $1 \mathrm{st}, 2 \mathrm{nd}, 3 \mathrm{rd}, 4 \mathrm{th}$, and 5 th dendritic orders. Dendritic branches leaving the cell body were defined as first order, while those which branched from the former were considered second order, and so on. The dendritic arborization was measured by placing the cell body and primary dendrites at the center of the first of a series of seven concentric rings (spaced at $40-\mu \mathrm{m}$ intervals) and counting all dendritic intersections with larger individual rings [28]. Additionally, the cross sectional area of PBN neuron perikarya was measured over the course of the experiment.

In all cases neuronal measurements were obtained at a magnification of X 400 using an image digitizing system (Perception Analysis System by Human-Computer Interface, Cambridge, UK). No attempt was made to correct for compression of the three-dimensional dendritic arbor to a two-dimensional sketch, since the relative differences between neurons remain constant when transformed from three to two dimensions [29]. Moreover, because the dendritic arbor is confined to the tissue section, no stereo- 
logical method was used. Additionally, for the soma parameters the image analyzer did some calculations similar to those previously described.

\subsection{Statistics}

Separate sets of statistical analyses were used to compare the score differences among ages and dietary conditions: 1) scores for body and wet brain weight were compared using a two-way ANOVA, 2 (nutritional conditions) $\times 3$ (ages); 2) the role of undernutrition on the dendritic order and dendritic crossings of branches during development was analyzed by using a three-way ANOVA, 2 (nutritional conditions) $\times 3$ (ages) $\times 5$ (dendritic orders), or 7 (concentric rings). To detect cumulative effects of undernutrition on the two dendritic measurements, the effects of the diet on all dendritic arbours at various ages, and the total number of dendritic orders or dendritic crossings, a two-way ANOVA was used, 2 (nutritional conditions) $\times 3$ (ages). The statistical differences between experimental groups were compared using the Fisher post hoc test. The threshold level of significance was set at $\mathrm{p} \leq$ 0.05 .

\section{RESULTS}

\subsection{Physical Growth Effects}

The UG body weight of animals was significantly reduced compared with those of CG subjects $(F(1,36)=$ 429.39; $\mathrm{p}<0.0001)$, and it was also affected by age $(\mathrm{F}(2,36)=702.74 ; \mathrm{p}<0.0001)$; there was a significant interaction between the diet by age $(\mathrm{F}(2,36)=79.75 ; \mathrm{p}<$ $0.0001)$. Post hoc comparisons showed significantly lower body weights in the UG $(p<0.001)$ at 12,20 , and 30 days than in the corresponding controls (Table 1). Additionally, brain weight comparisons indicated significantly lower values for UG compared to those of the CG subjects $(\mathrm{F}(1,36)=61.39 ; \mathrm{p}<0.0001)$; body weight was modified by age $(\mathrm{F}(2,36)=1250.36 ; \mathrm{p}<0.001)$, with no significant interactions between factors. Post hoc comparisons indicated lower brain wet weight values $(\mathrm{p}<$ 0.001) in the UG rats at all ages tested (Table 1).

\subsection{Dendritic Tree Effects}

The ANOVA comparisons of the dendritic orders between groups yielded significant reductions associated with diet $(\mathrm{F}(1,114)=11.89 ; \mathrm{p}<0.0007)$, and age $(2,114)=5.84 ; \mathrm{p}$ $<0.003)$, without interaction between factors. There were differences between the different orders (5) of dendritic complexity $(F(4,456)=394.05 ; p<0.0001)$, with an interaction between dendritic order by diet, $(\mathrm{F}(4,456)=5.51$; $\mathrm{p}<0.0002)$ and an interaction between dendritic order by age $(F(8,456)=1.99 ; p<0.046)$ when this factor was compared between groups. There was a total effect associated with diet, $(\mathrm{F}(1,114)=55.65 ; \mathrm{p}<0.0001$ and with

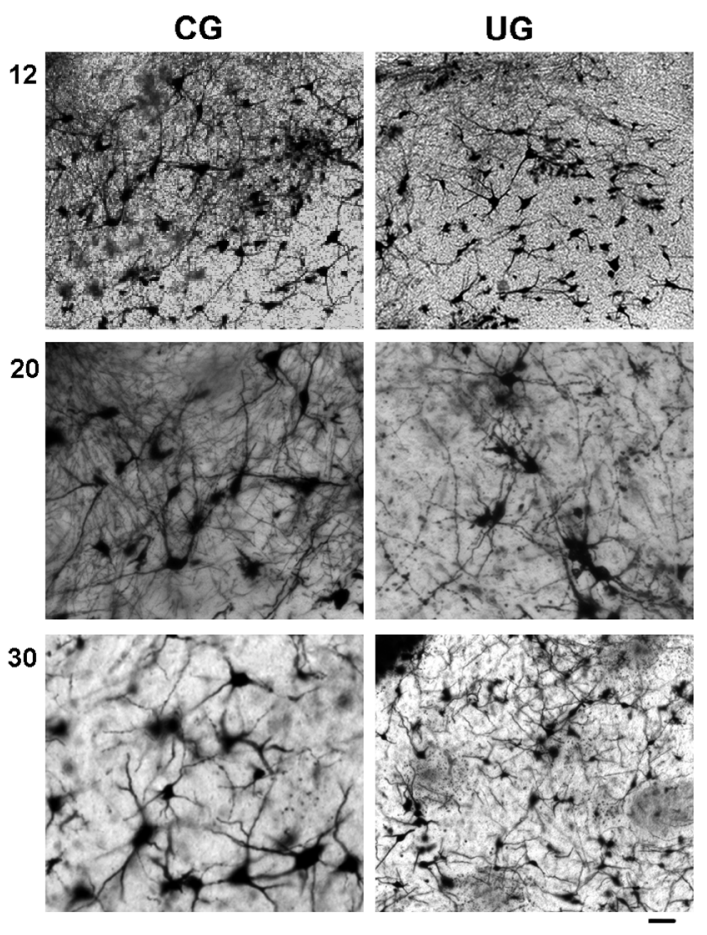

Figure 1. Photomicrographs of $\mathrm{PBN}$ multipolar neurons from CG and UG at 12, 20, and 30 days of age. Note the rich dendritic arbor in the $\mathrm{CG}$ and the density reduction in the dendritic neuropile of the UG rats. Bar $100 \mu \mathrm{m}$.

Table 1. Mean values \pm SEM of body and brain weight $(\mathrm{g})$ in $\mathrm{CG}$ and UG rats during development.

\begin{tabular}{cccccc}
\hline Age (days) & \multicolumn{2}{c}{ Body weight } & \multicolumn{2}{c}{ Brain weight } \\
\hline & \multicolumn{2}{c}{ CG } & UG & CG & UG \\
12 & $25.71 \pm 0.40$ & $15.12 \pm 0.49^{*}$ & $1.25 \pm 0.01$ & $1.20 \pm 0.00^{*}$ \\
20 & $34.35 \pm 0.48$ & $19.71 \pm 2.07^{*}$ & $1.37 \pm 0.01$ & $1.34 \pm 0.01^{*}$ \\
30 & $86.25 \pm 1.82$ & $45.25 \pm 1.40^{*}$ & $1.78 \pm 0.01$ & $1.73 \pm 0.00^{*}$ \\
Total & $48.75 \pm 6.00$ & $26.70 \pm 3.07^{*}$ & $1.50 \pm 0.05$ & $1.42 \pm 0.05^{*}$ \\
Factors & df & F & p $<$ & F & p $<$ \\
(A) Nutrition & 1.36 & 429.393 & 0.0001 & 61.39 & 0.0001 \\
(B) Age & 2.36 & 702.747 & 0.0001 & 1250.36 & 0.0001 \\
A $\times$ B & 2.36 & 79.758 & 0.0001 & 2.61 & NS \\
\hline
\end{tabular}

${ }^{*} \mathrm{p} \leq 0.05$, LSD Fisher test. NS, non-significant values.

age, $(\mathrm{F}(2,114)=4.57 ; \mathrm{p}<0.012)$. Post hoc comparisons at each developmental age showed that neurons of the UG animals consistently exhibited significant reductions $(p<0.05)$ in the number of branches, mainly in the middle and distal portions of the dendrites, when compared with CG rats. Thus, in the UG rats the branches of dendritic order 3 and 4 were reduced at 12 days of age, and those of 
orders 3 at 20 days of age, without significant effects at 30 days postnatally (Figure 2(a)).

The density of the dendritic branches of PBN neuron, taken as the number of crossings of dendrites per circle indicated significant reductions in the dendritic density of neurons in the UG subjects compared to the CG animals $(\mathrm{F}(1,114)=55.65 ; \mathrm{p}<0.0001)$; and a significant effect of age $(F(2,114)=4.57 ; p<0.01)$. No interaction between factors was obtained. When the comparison was done based on the circle crossings, significant differences were observed $(F(6,684)=559.49 ; p<0.0001)$. The interactions between the circle (length) and the dietary treatment $(\mathrm{F}(6,684)=3.765, \mathrm{p}<0.001$ as well as between and the length and the age $(F(12,684)=3.47 ; \mathrm{p}<0.0001)$. There was a total effect associated with the diet, $(1,114)$ $=55.65 ; \mathrm{p}<0.0001$, and age, $(\mathrm{F}(2,114)=4.57 ; \mathrm{p}<0.012)$. The post hoc comparisons at each developmental age indicated significant score reductions $(p<0.05)$ in the dendritic crossings of the UG animals at postnatal days 12 , and 20 on circles $1,2,3$, and 4 . Furthermore, a significant reduction in the dendritic crossing of the UG animals at 30 day of age only on circle 5 was obtained. These dendritic density reductions in the UG subjects were particularly affected the more proximal and middle portions of the PBN neuronal dendritic tree (Figure 2(b)). The cumulative effects of undernutrition on the number of dendrites and the density of branches along the ages indicated significant reduced values $(p<0.05)$ in both UG neuronal measurements along the ages (Figures 2(a) and (b)).

\subsection{Perikarya Effects}

The ANOVA comparisons of the perikarya scores indicated significant reductions of the neuron cross sectional area in the UG subjects $(\mathrm{F}(1,114)=12.95 ; \mathrm{p}<0.0004)$ with no significant effects of age or interactions between factors. The post hoc Fisher test showed significant reductions $(\mathrm{p}<0.05)$ of cross sectional areas in the UG at 20 and 30 days of age (Figure 3 ). In the CG subjects the values of the cross sectional area of perikarya did not change with age. However, in the UG there was a slow reduction in these values from 12 to 30 postnatal days (Figure 3). The cumulative effects of undernutrition on the cross sectional area values with ages yielded a significant reduction $(\mathrm{p}<0.05)$ in the UG neuronal measurements along the ages (Figure 3).

\section{DISCUSSION}

The current findings indicated that, at all the ages studied body and brain weights were consistently reduced in the UG subjects when compared to their corresponding CG rats, and they were in agreement with previous studies
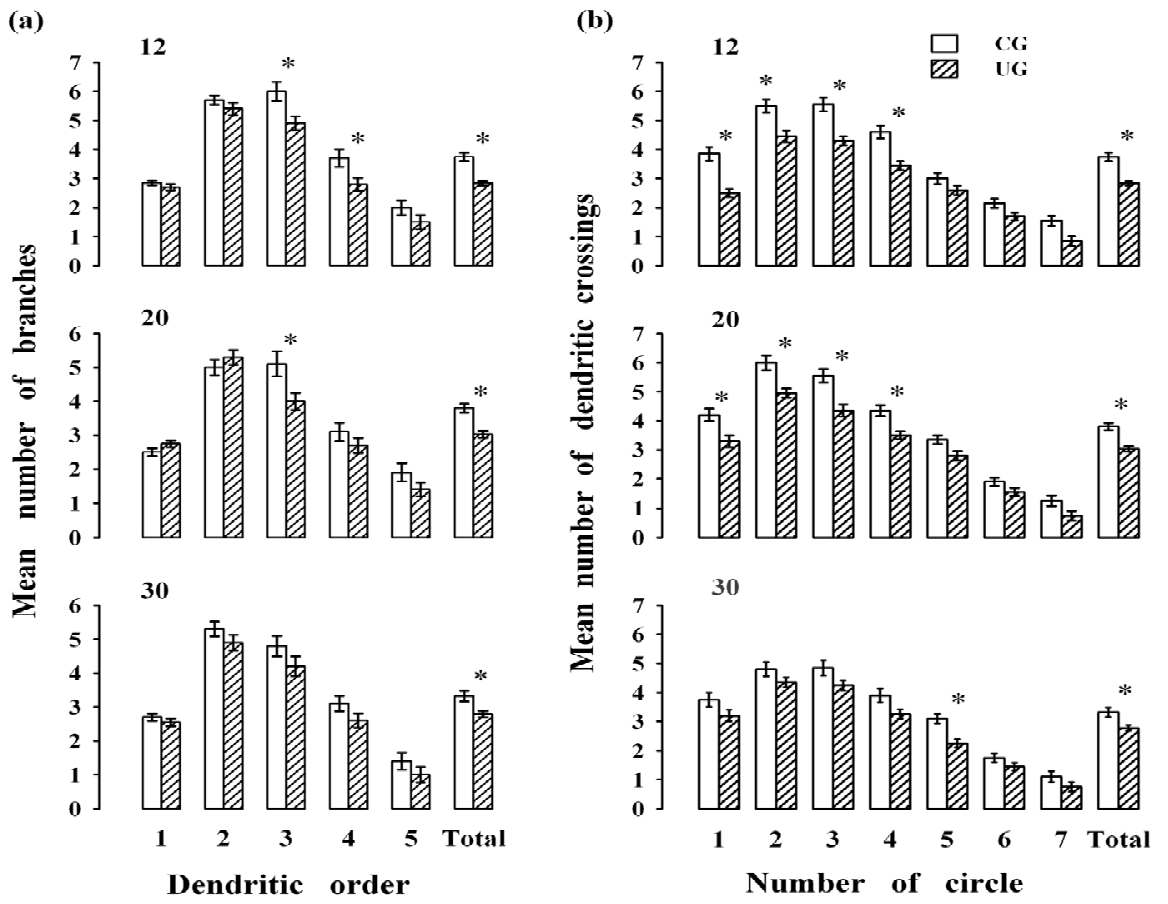

Figure 2. Mean values \pm SEM for the (a) dendritic branches of different orders and (b) dendritic intersections of multipolar neurons $(n=20$ /age group) at different ages and under different experimental conditions. Note, a reduction in the number of branches and their crossings at most ages of UG. *Post hoc statistical differences between groups, $p<0.05$. 
showing that pre- and neonatal undernourishment interferes with the physical development, huddling of pups, and ear- and eye-opening and delayed sensory maturation including the development of olfactory and gustatory pathways in newborn rats $[21,30,31]$.

The impoverishment in the dendritic tree may provoke a disruption in the interactions of the asynchronous afferent messages arising from different CNS sources (central amygdala, insular cortex, lateral hypothalamus, and bed nucleus of the stria terminalis), and a distortion both in time and location through a deficient hypoplastic neuronal network associated with the early undernourishment [19-22]. Furthermore, if different neuronal patterns of information organized in space and time seem to reflect the coding of the quality of taste, then the PBN neurons may be out of tune and therefore unable to generate the appropriate integrative responses to the limbic structures and to participate in generating the sensory and hedonic aspects of gustatory stimuli [8,32-34].

A point of interest derived from the current morphological alterations elicited by early undernutrition concerns the possible effects on PBN neuronal development and their impact upon the plasticity taking place at the synaptic loci of the dendritic tree, which may affect the integration of codes at other gustatory relay levels. Thus, our data indicated that in the UG rats, the number of dendrites in the middle and distal portions of the dendritic tree was significantly reduced; meanwhile, the dendritic density was diminished at the proximal and middle portions of the dendritic tree, and these reductions persist when the cumulative effects were evaluated along the ages. Furthermore, on postnatal day 30 the minimal reduction in the number and density of dendritic branches of the UG subjects may be associated with the 6-day period of dietary rehabilitation, although the reduced neu-

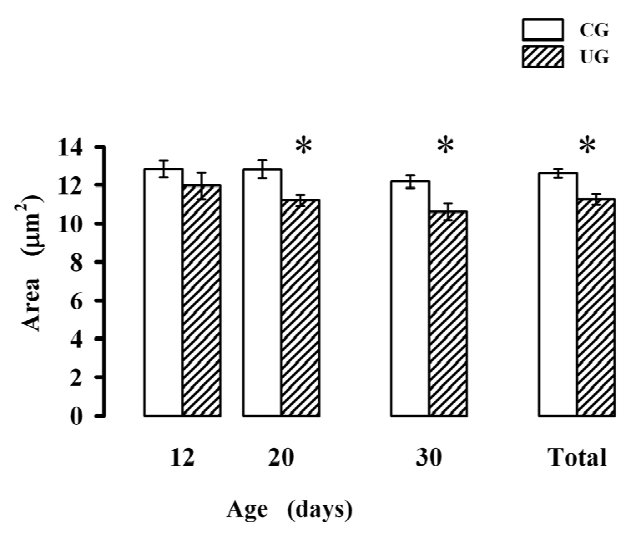

Figure 3. Mean values \pm SEM of cross sectional parikarya area of multipolar PBN neurons of CG and UG subjects during development. ${ }^{*}$ Significant difference between groups $\mathrm{p}$ $<0.05$ for each particular age obtained with the Fisher LSD test. ronal cross sectional area in the UG values was not recovered. According to previous electrophysiological studies, the location of the dendritic synapse (distal or proximal) may significantly and differentially affect the amplitude and kinetics of the responses, as well as the propagation characteristics of the action potentials; later, these changes may partly disrupt the elaboration of synaptic plasticity and affect both the sensory and the hedonic aspects of tastants [8]. These alterations may play a role in the development of complex physiological phenomena such as food intake, taste discrimination, taste aversion learning and appetitive behavior as previously described in PBN lesions and perinatally undernourished rats [34-42].

The cross sectional area reductions in the PBN perikarya and other types of neurons are at present controversial; they depend on the locations of the neurons within the central, autonomic and peripheral neuronal systems [21,43-45] and their age [46]. Furthermore, they may be reflecting a diminished release of neurotransmitters from the presynaptic endings or reductions in the number of axosomatic synapses, because of a possible sensory deprivation, chronic exposure to underfeeding stress, or cellular/biochemical changes induced by energy deprivation mechanisms commonly associated with the undernourishment paradigm [36,45,47-52]. However, analyses of different neuronal types and PBN locations, of the influence of sensory and autonomic inputs, and with different methodological approaches such as overfeeding are required to understand the role of this complex brain structure in the mechanisms underlying normal food intake.

\section{ACKNOWLEDGEMENTS}

This work was partly supported by DGAPA/UNAM, IN207307-3. We thank Dr Dorothy D. Pless for editorial assistance, N. Hernandez for neuronal image reconstruction, and A. Martin for collecting data.

\section{REFERENCES}

[1] Hermann, G.E. and Rogers, R.C. (1985) Convergence of vagal and gustatory afferent input within the parabrachial nucleus of the rat. Journal of the Autonomic Nervous System, 13, 1-7. doi:10.1016/0165-1838(85)90002-5

[2] Kobashi, M. and Adachi, A. (1986) Projection of nucleus tractus solitarius units influenced by hepatoportal afferent signal to parabrachial nucleus. Journal of the Autonomic Nervous System, 16, 153-158.

[3] Moga, M.M., Herbert, H., Hurley, K.M., et al. (1990) Organization of cortical, basal forebrain, and hypothalamic afferents to the parabrachial nucleus in the rat. Journal of Comparative Neurology, 295, 624-661. doi:10.1002/cne.902950408

[4] Hanamori, T. and Ishiko, N. (1993) Cardiovascular responses to gustatory and mechanical stimulation of the nasopharynx in rats. Brain Research, 619, 214-222. 


\section{doi:10.1016/0006-8993(93)91614-X}

[5] Yamamoto, T., Shimura, T., Sakai, N., et al. (1994) Representation of hedonics and quality of taste stimuli in the parabrachial nucleus of the rat. Physiology and Behavior, 56, 1197-1202. doi:10.1016/0031-9384(94)90366-2

[6] Mizusawa, A., Ogawa, H., Kikuchi, Y., et al. (1995) Role of the parabrachial nucleus in ventilatory responses of awake rats. Journal of Physiology, 489, 877-884.

[7] Shimura, T., Tokita, K. and Yamamoto, T. (2002) Parabrachial unit activities after the acquisition of conditioned taste aversion to a non-preferred HCL solution in rats. Chemical Senses, 27, 153-158. doi:10.1093/chemse/27.2.153

[8] Sewards, T.V. (2004) Dual separate pathways for sensory and hedonic aspects of taste. Brain Research Bulletin, 62, 271-283.

[9] Haino, T., Hironaka, S., Ooka, T., et al. (2010) Orosensory deprivation alters taste-elicited c-Fos expression in the parabrachial nucleus of neonatal rats. Neuroscience Research, 67, 228-235. doi:10.1016/j.neures.2010.03.007

[10] Coizet, V., Dommett, E.J., Klop, E.M., et al. (2010) The parabrachial nucleus is a critical link in the transmission of short latency nociceptive information to midbrain dopaminergic neurons. Neuroscience, 168, 263-272. doi:10.1016/j.neuroscience.2010.03.049

[11] Callison, D.A. and Spencer, J.W. (1968) Effect of chronic undernutrition and/or visual deprivation upon the visual evoked potential from the developing rat brain. Developmental Psychobiology, 1, 196-204. doi:10.1002/dev.420010308

[12] Salas, M. and Cintra, L. (1973) Nutritional influences upon somatosensory evoked responses during development in the rat. Physiology and Behavior, 10, 1019-1022. doi:10.1016/0031-9384(73)90182-0

[13] Math, F. and Davrainville, J.L. (1980) Eletrophysiological study of the postnatal development of mitral cell activity in the rat olfactory bulb. Influence of undernutrition. Brain Research, 194, 223-227. doi:10.1016/0006-8993(80)91333-5

[14] Salas, M., Torrero, C., Regalado, M., et al. (1994) Dendritic arbor alterations in the medial superior olivary neurons of neonatally underfed rats. Acta Anatomica, 151, 180-187. doi:10.1159/000147661

[15] Frias, C., Torrero, C., Regalado, M., et al. (2009) Development of mitral cells and olfactory bulb layers in neonatally undernourished rats. Nutritional Neuroscience, 12, 96-104. doi:10.1179/147683009X423238

[16] Cragg, B.G. (1972) The development of cortical synapses during starvation in the rat. Brain, 95, 143-150. doi:10.1093/brain/95.1.143

[17] Salas, M., Diaz, S. and Nieto, A. (1974) Effects of neonatal food deprivation on cortical spines and dendritic development of the rat. Brain Research, 73, 139-144. doi:10.1016/0006-8993(74)91012-9

[18] McConell, P. and Berry, M. (1978) The effect of refeeding after neonatal starvation on Purkinje cell dendritic growth in the rat. Journal of Comparative Neurology, 178, 759-772. doi:10.1002/cne.901780410
[19] Escobar, C. and Salas, M. (1993) Neonatal undernutrition and amygdaloid nuclear complex development: An experimental study in the rat. Experimental Neurology, 122, 311-318. doi:10.1006/exnr.1993.1130

[20] Andrade, J.P., Castanheira-Vale, A.J., Paz-Dias, P.G., et al. (1996) The dendritic trees of neurons from the hippocampal formation of protein-deprived adult rats. A quantitative Golgi study. Experimental Brain Research, 109, 419-433. doi:10.1007/BF00229626

[21] Rubio, L., Torrero, C., Regalado, M., et al. (2004) Alterations in the solitary tract nucleus of the rat following perinatal food restriction and subsequent nutritional rehabilitation. Nutritional Neuroscience, 7, 291-300. doi:10.1080/10284150400019922

[22] Salas, M., Torrero, C., Rubio, L., et al. (2012) Effects of perinatal undernutrition on the development of neurons in the insular cortex. Nutritional Neuroscience, 15, 20-25. doi:10.1179/1476830512Y.0000000014

[23] Vanderbergh, J.G. (2003) Prenatal exposure and sexual variation. American Scientist, 91, 218-225. doi:10.1511/2003.3.218

[24] Altman, J. and Bayer S.A. (1995) Atlas of prenatal rat brain development. CRC Press, Boca Raton.

[25] Lynch, A. (1976) Postnatal undernutrition: An alternative method. Developmental Psychobiology, 9, 39-48. doi:10.1002/dev.420090107

[26] Salas, M., Torrero, C. and Pulido, S. (1984) Long-term alterations in the maternal behavior of neonatally undernourished rats. Physiology and Behavior, 33, 273-278. doi:10.1016/0031-9384(84)90111-2

[27] Paxinos, G. and Watson, C. (1986) The rat brain in stereotaxic coordinates. Academic Press Inc., San Diego.

[28] Sholl, D.A. (1956) The organization of the cerebral cortex. Halfner, New York.

[29] Spinelli, D.N., Jensen, F.E. and Di Prisco, G.V. (1980) Early experience effect on dendritic branching in normally reared kittens. Experimental Neurology, 68, 1-11. doi:10.1016/0014-4886(80)90063-1

[30] Soriano, O., Regalado, M., Torrero, C., et al. (2006) Contributions of undernutrition and handling to huddling development of rats. Physiology and Behavior, 89, 543-551.

[31] Frias, C., Torrero, C., Regalado, M., et al. (2006) Organization of olfactory glomeruli in neonatally undernourished rats. Nutritional Neuroscience, 9, 49-55. doi:10.1080/10284150500506042

[32] Magee, J.C. and Cook, E.P. (2000) Somatic EPSP amplitude is independent of synapse location in hippocampal pyramidal neurons. Nature Neuroscience, 3, 895-903. doi: $10.1038 / 78800$

[33] Williams, S.R. and Stuart, G.J. (2000) Back propagation of physiological spike trains in neocortical pyramidal neurons: Implications for temporal coding in dendrites. Journal of Neuroscience, 20, 8238-8246.

[34] Peciña, S., Smith, K.S. and Berridge, K.C. (2006) Hedonic hot spots in the brain. The Neuroscientist, 12, 500511. doi: $10.1177 / 1073858406293154$

[35] Escobar, C. and Salas, M. (1995) Dendritic branching of 
claustral neurons in neonatally undernourished rats. Biology of the Neonate, 68, 47-54. doi:10.1159/000244217

[36] Saggu, S. and Lundy, R. (2008) Forebrain neurons that project to the gustatory parabrachial nucleus in rat lack glutamic acid decarboxylase. American Journal of Physiology Regulatory Integrative Comparative Physiology, 294, R52-R57. doi:10.1152/ajpregu.00635.2007

[37] De la Torre, M.L. and Agüero, A. (2008) The role of the dorsal-most part of the lateral parabrachial nucleus in the processing of hypertonic $\mathrm{NaCl}$ using different conditioned flavor avoidance paradigms. Experimental Brain Research, 186, 481-491. doi:10.1007/s00221-007-1250-3

[38] Hajnal, A., Norgren, R. and Kovacs, P. (2009) Parabrachial coding of sapid sucrose: Relevance to reward and obesity. Annals of the New York Academy of Science, 1170, 347364. doi:10.1111/j.1749-6632.2009.03930.x

[39] Froemke, R.C., Letzkus, J.J., Kampa, B.M., et al. (2010) Dendritic synapse location and neocortical spike-timingdependent plasticity. Frontiers in Synaptic Neuroscience, 2, 1-14. doi:10.3389/fnsyn.2010.00029

[40] Hill, D.L. and Almli, C.R. (1983) Parabrachial nuclei damage in infant rats produces residual deficits in gustatory preferences/aversions and sodium appetite. Developmental Psychobiology, 16, 519-533.

[41] Yamamoto T. (1993) Neural mechanisms of taste aversion learning. Neuroscience Research, 16, 181-185. doi:10.1016/0168-0102(93)90122-7

[42] Dayawansa, S., Peckins, S., Ruch, S. et al. (2011) Parabrachial and hypothalamic interaction in sodium appetite. American Journal Regulatory Integrative Comparative Physiology, 300, R1091-R1099. doi:10.1152/ajpregu.00615.2010

[43] Bhide, P.G. and Bedi, K.S. (1984) The effects of environmental diversity on well fed and previously undernourished rats: Neuronal and glial cell measurements in the visual cortex (area 17). Journal of Anatomy, 138, 447461.

[44] Castelucci, P., Souza, R.R., Angelis, R.C., et al. (2002) Effects of pre-and postnatal protein deprivation and post- natal refeeding on myenteric neurons of the rat large intestine: A quantitative morphological study. Cell Tissue Research, 310, 1-7. doi:10.1007/s00441-002-0615-y

[45] Torrero, C., Regalado, M., Perez, E., et al. (2005) Neonatal food restriction and binaural ear occlusion interfere with the maturation of cortical motor pyramids in the rat. Nutritional Neuroscience, 8, 63-66. doi:10.1080/10284150400027131

[46] Altavista, M.C., Rossi, P., Bentivoglio, A.R., et al. (1990) Aging is associated with a diffuse impairment of forebrain cholinergic neurons, Brain Research, 508, 51-59. doi:10.1016/0006-8993(90)91116-X

[47] Reddy, T.S. and Horrocks L.A. (1986) Effects of neonatal undernutrition of rats on the synthesis of phosphatidylcholine and phosphatidylethanolamine by microsomes from gray matter and white matter. International Journal of Developmental Neuroscience, 4, 89-95. doi:10.1016/0736-5748(86)90020-1

[48] Hamori, J. (1990) Morphological plasticity of postsynaptic neurones in reactive synaptogenesis. Journal of Experimental Biology, 153, 251-260.

[49] Watanabe, Y., Gould, E. and McEwen, B.S. (1992) Stress induces atrophy of apical dendrites of hippocampal CA3 pyramidal neurons. Brain Research, 588, 341-345. doi:10.1016/0006-8993(92)91597-8

[50] Sakai, N. and Yamamoto, T. (1998) Role of the medial and lateral parabrachial nucleus in acquisition and retention of conditioned taste aversion in rats. Behavioural Brain Research, 93, 63-70.

[51] Simões, L.S., Boldrini, S.C., Binotti, C.B., et al. (2009) Impact of pre and postnatal protein energy deprivation on structure of the trigeminal ganglion of weanling rats. The Open Nutrition Journal, 3, 1-4. doi:10.2174/1874288200903010001

[52] Gomes, P.S., Nyengaard, J.R., Misawa, R., et al. (2009) Atrophy and neuron loss: effects of a protein-deficient diet on sympathetic neurons. Journal of Neuroscience Research, 87, 3568-3575. doi:10.1002/jnr.22167 